\title{
CONFLICTO Y ESTRATEGIA SOCIAL EN LA MINERÍA Y LOS HIDROCARBUROS PERUANOS: LOS USOS VARIADOS DE LA PARTICIPACIÓN EN LA EVALUACIÓN DE IMPACTO AMBIENTAL*
}

\section{Conflict and Social Strategy in Peruvian Mining and Hydrocarbons: The Varied Uses of Participation in Environmental Impact Assessment}

\author{
MAIAH JASKOSKI
}

Northern Arizona University, Estados Unidos

\begin{abstract}
RESUMEN
Este artículo examina cómo las comunidades locales movilizadas contra la extracción utilizan la audiencia pública que es parte de la evaluación de impacto ambiental (EIA). En base al análisis de trece conflictos importantes sobre hidrocarburos y minería en Perú, se observa que en algunos casos las comunidades actuaron dentro del espacio participativo provisto, mientras que en otros casos se organizaron "en torno" o "en reacción" al mismo, utilizando los procedimientos institucionales como puntos focales para la escalada del conflicto. El análisis explica la variación en las tácticas comunitarias frente al EIA subrayando la importancia de: (1) la fase del proyecto de extracción en la que se desarrolló el conflicto, (2) la definición-o no-de la comunidad como suficientemente afectada por parte del EIA, mereciendo, por lo tanto, su inclusión en la audiencia, y (3) el grado de cohesión de las comunidades incluidas.
\end{abstract}

Palabras claves: evaluación de impacto ambiental, instituciones participativas, Perú, conflicto por recursos.

\begin{abstract}
This article examines how local communities mobilized against extraction use the public hearing that is part of environmental impact assessment. Based on analysis of thirteen important hydrocarbon and mining conflicts in Peru, it observes that in some cases communities acted within the provided participatory space, whereas in other cases they organized "around" or "in reaction" to it, using the institutional procedures as focal points for escalating conflict. The study explains variation in community uses of the hearing using three variables: the stage of planned extraction at the time that conflict broke out; whether the project design placed mobilized actors inside or outside of the project's area of direct impact; and the degree of unity among the "insiders."
\end{abstract}

Keywords: environmental impact assessment, participatory institutions, Peru, resource conflict.

\footnotetext{
Agradecimientos: Se agradece a los individuos en Perú que compartieron sus conocimientos sobre el conflicto extractivo y los reglamentos ambientales; a Candelaria Garay y tres revisoras anónimos de Revista de Ciencia Política por sus comentarios en profundidad sobre el manuscrito; y a Moisés Arce, Kent Eaton, Veronica Herrera, Kathy Hochstetler, Lindsay Mayka, y Thea Riofrancos por sus comentarios sobre la versión preliminar del análisis. Los viajes de trabajo de campo para la investigación fueron financiados por Northern Arizona University y el U.S. Department of Defense Threat Reduction Agency Advanced Systems and Concepts Office.
} 


\section{INTRODUCCIÓN}

La nueva producción minera y de hidrocarburos en América Latina es de gran importancia para las empresas, el estado, y las comunidades locales en las regiones en que se produce la extracción. Estas industrias crecieron drásticamente entre 1990 y 2010, acompañando el incremento de los precios de las materias primas y la adopción de políticas económicas liberales que alentaron la inversión privada. Esta expansión hizo que los inversores privados y los gobiernos de la región se vieran cada vez más comprometidos con la extracción. Al mismo tiempo, la expansión de la minería y los hidrocarburos generó preocupación en las comunidades locales por el impacto ambiental, cultural y social de la exploración y por la distribución de las ganancias producidas por la extracción. Al enfrentar nuevos proyectos extractivos, algunas comunidades afectadas se movilizaron para impedirlos, mientras que otras exigieron ser compensadas por el impacto adverso de estos proyectos, obteniendo de este modo una proporción de las rentas provenientes de la extracción (De Echave et al. 2009; Arellano Yanguas 2011; Bebbington y Bury 2013; Arce 2014; Ponce y McClintock 2014; Henao y González Espinosa 2016; Delamaza et al. 2017; Pellegrini y Arsel 2018).

Las comunidades afectadas por las industrias extractivas cuentan con diferentes canales para expresar sus preocupaciones sobre nuevos proyectos extractivos. Uno de estos canales es el estudio de impacto ambiental (EIA), que se generalizó en toda la región en los años noventa con la adopción de leyes y regulaciones ambientales. El EIA es un documento producido por consultores privados contratados por quienes lideran un proyecto extractivo con el fin de identificar los posibles impactos ocasionados por dicho proyecto y las medidas necesarias para mitigarlos. El estado debe informar a las comunidades locales sobre el proyecto en cuestión, a menudo en audiencias públicas. Además, en América Latina y el Caribe se encuentran quince de los veintidós gobiernos que han ratificado la Convención de Pueblos Indígenas y Tribales de 1989 de la Organización Internacional del Trabajo (OIT 169). La OIT 169 exige que el estado consulte con los pueblos nativos afectados por el nuevo desarrollo antes de aprobarlo. Este derecho a la "consulta previa" está regulado a nivel nacional en varios países latinoamericanos.

Este artículo analiza el papel de las audiencias públicas del EIA en los conflictos en torno a nuevos proyectos de extracción. Para ello, examina trece conflictos mineros y de hidrocarburos de gran relevancia en Perú desde principios de la década de 2000. Si bien en algunas comunidades afectadas, la sociedad civil se encontraba organizada y participó activamente en las audiencias, en otros casos las comunidades se organizaron "alrededor de" o "en reacción a" las audiencias, utilizando esta institución como punto focal para escalar el conflicto. En estos casos, las comunidades bloquearon la audiencia; impugnaron su exclusión de la audiencia; o buscaron impedir la extracción aun antes de que se estableciera la etapa de audiencia, centrando sus reclamos en el derecho legal al uso de las tierras. El análisis explica la variación en las tácticas comunitarias 
frente al EIA subrayando la importancia de: (1) la fase del proyecto de extracción en la que se desarrolló el conflicto, (2) la definición-o no-de la comunidad como suficientemente afectada por parte del EIA, mereciendo, por lo tanto, su inclusión en la audiencia, y (3) el grado de cohesión de las comunidades incluidas.

Esta explicación de por qué en algunos casos la audiencia pública dirija el conflicto, mientras que en otros casos lo exacerba, contribuye a la literatura sobre la participación ciudadana y el conflicto en las industrias extractivas. Este cuerpo de investigación no ha profundizado sistemáticamente sobre el modo en que las instituciones participativas en el desarrollo extractivo otorgan indirectamente voz a las comunidades, creando oportunidades para que los ciudadanos se organicen en torno a espacios participativos.

En línea con varios estudios y compilaciones de investigaciones, este trabajo analiza conflictos mineros e hidrocarburíferos (véase por ejemplo Scurrah 2008; Bebbington y Bury 2013; Arce 2014; Thorp et al. 2014). Esta decisión tiene sentido si consideramos que el propósito de la audiencia en los dos sectores es el mismo: informar a las comunidades sobre el proyecto de uso del subsuelo, que pertenece al estado central. Además, las comunidades a menudo emplean tácticas parecidas en conflictos extractivos en la minería y los hidrocarburos. ${ }^{1} \mathrm{La}$ elección analítica también posibilita el desarrollo de un marco causal general que se aplique a los dos sectores. Sin embargo, vale la pena señalar que hay diferencias estructurales importantes entre la minería y los hidrocarburos y que, en algunos casos, estas diferencias pueden requerir de un tratamiento separado de cada sector para entender mejor el conflicto extractivo.

\section{PARTICIPACIÓN Y CONFLICTO EN LAS INDUSTRIAS EXTRACTIVAS}

Uno de los principales enfoques en la literatura sobre el conflicto social en la extracción se centra en cómo las comunidades ejercen presión en los espacios participativos formales previstos por la consulta previa y las audiencias públicas del EIA. Otros estudios analizan la movilización en torno o en reacción a las oportunidades de participación existentes. Sin embargo, no hay estudios que busquen identificar las condiciones bajo las cuales las comunidades actúan en los espacios provistos por las instituciones participativas o, por el contrario, se

\footnotetext{
En Colombia se usa el sistema de justicia para resolver conflictos tanto en el sector hidrocarburífero como en la minería (Jaskoski 2020). Los bloqueos se construyen para interrumpir el desarrollo en los dos sectores también; por ejemplo, en el caso petrolero en Putumayo, Colombia (Jaskoski 2020: 548), y en el conflicto minero peruano de Las Bambas [2], que se discute en el presente estudio. Se ven las protestas en la minería, como se describe en casos de esta investigación, y también en los hidrocarburos: en el conflicto sobre el Lote 56 de Camisea, 2.500 Machiguenga participaron en una huelga en reacción a roturas de tubería entre agosto y noviembre de 2005, después de los eventos descritos abajo (Ross 2008: 251-52).
} 
movilizan en torno a estos espacios. La literatura tampoco explora la variación en la movilización por fuera de los espacios participativos existentes. ${ }^{2}$

Es posible que incluso la oportunidad de participar fomente la movilización en las instancias participativas. Esta es la conclusión, por ejemplo, del análisis interregional de conflictos en proyectos de oleoductos de hidrocarburos de McAdam et al. (2010). Otros estudios postulan que las características de las comunidades, el diseño institucional y el apoyo de aliados inyectan un significado real a procedimientos que de otro modo estarían vacíos de sustancia. Aquellas comunidades que tienen mayor fuerza organizativa (Schilling-Vacaflor 2013: 209-214; Schilling-Vacaflor y Flemmer 2015: 835) y que cuentan con aliados-por ejemplo, intelectuales (Devlin y Yap 2008) u organizaciones no gubernamentales (ONGs) internacionales (Schilling-Vacaflor y Flemmer 2015: 833) - han mostrado mayor cohesión e influencia. ${ }^{3}$

Cuando se considera a los aliados de las comunidades afectadas, el estado entra en escena: Kathryn Hochstetler identifica el papel del Ministerio Público brasileño en las "coaliciones de bloqueo" de proyectos energéticos, un rol que incluye asegurar la implementación correcta de los procedimientos de las audiencias públicas del EIA (Hochstetler 2011: 355, 357). En Perú, la Defensoría del Pueblo ha apoyado a las comunidades indígenas en las consultas previas (Schilling-Vacaflor y Flemmer 2015: 833).

Algunos elementos del diseño institucional también parecen mejorar tanto la calidad de la participación como la influencia de las comunidades afectadas a través de la participación, por lo menos en la consulta previa. En su extensa investigación sobre Bolivia y Perú, Riccarda Flemmer y Almut Schilling-Vacaflor encuentran que ciertos atributos institucionales fortalecen a las comunidades en los procesos de consulta-características que se corresponden con el énfasis de la literatura en la cohesión comunal y el apoyo estatal descrito arriba. En particular, las autoras mantienen que las consultas deben ser lideradas por una dependencia estatal imparcial, ${ }^{4}$ realizarse antes de que se diseñe un proyecto en detalle (Schilling-Vacaflor 2013: 209), ser planificadas con la participación de las comunidades, ${ }^{5}$ incluir a los líderes indígenas regionales o nacionales (Schilling-Vacaflor y Flemmer 2015: 834) y producir acuerdos vinculantes (Schilling-Vacaflor y Flemmer 2015: 835-36). Los procesos de diseño también han influido sobre la calidad de la consulta previa: la participación indígena en la redacción de leyes y reglamentos de consulta previa ha inyectado legitimidad

\footnotetext{
Una excepción es Jaskoski (2020), que trata la consulta previa colombiana y que, junto con el presente artículo, forma parte de una agenda más amplia de investigación.

Devlin y Yap (2008) analiza el bloqueo de proyectos de gran escala en Brasil, Sudáfrica, Filipinas y Taiwán; Schilling-Vacaflor (2013) trata de la consulta previa boliviana; y Schilling-Vacaflor y Flemmer (2015) examina la consulta previa en Perú.

Schilling-Vacaflor (2013: 215); y Schilling-Vacaflor y Flemmer (2015: 832).

Schilling-Vacaflor y Flemmer (2015: 829); y Flemmer y Schilling-Vacaflor (2016: 177-78).
} 
a la institución, lo que a su vez ha generado una mayor participación por parte de las comunidades en las consultas. ${ }^{6}$

Otra parte de la literatura considera poco probable que las comunidades tengan vos en estas instituciones participativas. Desde esta perspectiva, las comunidades no adquieren poder por medio de la participación. Al contrario, estos estudios consideran que las instituciones de participación sirven para expedir la extracción. ${ }^{7}$

Sin embargo, algunos académicos que adoptan esta posición pesimista ven que, de forma menos directa, las instituciones sí pueden provocar la movilización. Enfrentados a instituciones diseñadas para desempeñar una función puramente informativa, los activistas en algunos casos han intentado impedir audiencias o consultas previas ${ }^{8}$ o han declarado ilegítimos a los procesos participativos ya concluidos. ${ }^{9}$ Las comunidades también se han movilizado para exigir que el estado siga procedimientos formales de participación (Leifsen et al. 2017). Las limitaciones que encuentran las comunidades para debatir e influir sobre el desarrollo de proyectos en el EIA chileno ha llevado a los actores involucrados en conflictos ambientales a usar otros mecanismos, por fuera de las instancias participativas existentes, para resolverlos (Delamaza 2010: 2425). Muchas veces los conflictos surgen justamente porque las comunidades afectadas no se sienten representadas en las estructuras participativas de la evaluación de impacto ambiental (O’Faircheallaigh 2017; Barandiarán y Rubiano-Galvis 2019). Estos conflictos también han tenido lugar en el desarrollo y la implementación de la consulta previa. ${ }^{10}$

En su conjunto, la investigación sobre la participación ciudadana y el conflicto social en las industrias extractivas identifica diferentes formas en que los grupos se movilizan en el seno y en torno de ellas. Sin embargo, no hay estudios que describan sistemáticamente las distintas maneras en que las comunidades emplean las instituciones participativas o que expliquen por qué los activistas se involucran en instancias participativas establecidas en algunos casos y no en

6 Véase Falleti y Riofrancos (2018) sobre los casos boliviano y ecuatoriano. Schilling-Vacaflor y Flemmer (2015) examina el caso negativo de la consulta previa peruana.

$7 \quad$ Véase por ejemplo Pellegrini y Ribera Arismendi (2012) y Eisenstadt y West (2019: capítulo 3) con respecto a la consulta previa en Bolivia y Ecuador, respectivamente. O'Faircheallaigh (2017) y Barandiarán y Rubiano-Galvis (2019) presentan esta perspectiva sobre la participación en el EIA, en general.

$8 \quad$ Para el análisis del uso de la audiencia pública en el EIA, véase Walter y Martinez-Alier (2010: 298), que estudian un proyecto de mina de oro a tajo abierto en Mendoza, Argentina; y Li (2015: capítulo 5) y Jaskoski (2014: 875-76), que analizan conflictos mineros peruanos. Para un análisis del mismo uso de la consulta previa en Colombia, véase Rodríguez-Garavito (2011: 296) y Jaskoski (2020: 545-47).

$9 \quad$ Para este empleo de las instituciones participativas, véase Jaskoski (2014: 876-77) sobre el caso peruano Conga, en que la institución relevante fue la audiencia de EIA, y Jaskoski (2020: 547-48) sobre un conflicto colombiano-en la mina Mandé Norte-en que las comunidades se movilizaron en torno a la consulta previa.

10 Dicho conflicto alrededor de la consulta previa se ha visto en Bolivia (D. Bebbington 2012; Schilling-Vacaflor 2017), Chile (Carcelén Pacheco y Mir Bennett 2014); Colombia (Orduz Salinas 2014); Ecuador (Leifsen et al. 2017; Riofrancos 2017); Guatemala (Elías y Sánchez 2014); y Perú (Schilling-Vacaflor y Flemmer 2015; Sanborn et al. 2016: 9, 21-22, 34-35). 
otros. El presente estudio persigue este objetivo, y a tal fin examina la participación y el conflicto social por nuevos proyectos extractivos en Perú.

\section{CONFLICTO EXTRACTIVO Y PARTICIPACIÓN EN LOS EIA EN PERÚ}

Perú provee un buen contexto para indagar y explicar la variedad en el uso de la audiencia pública en conflictos extractivos. La economía nacional depende profundamente de la extracción, lo que causa conflictos extractivos de gran envergadura. Además, la audiencia pública para el EIA está institucionalizada en el país.

La inversión en los hidrocarburos y la minería-que históricamente han sido sectores críticos de la economía peruana-aumentó en los años noventa y luego se disparó en la década de 2000 hasta la de 2010 (A. Bebbington 2010: 100; A. Bebbington et al. 2010: 309-311; CooperAcción 2018: 5). El crecimiento se dio en una coyuntura de política económica liberal en la que se promovió la inversión privada extranjera. Esta estrategia comenzó con el presidente Alberto Fujimori (1990-2000) (Bury y Norris 2013: 99-100) y continuó en los gobiernos posteriores. Es así que, en los dos primeros años de su (segunda) presidencia, el presidente Alan García (2006-2011) emitió casi cien decretos para alentar la inversión privada, incluso en la explotación de recursos naturales (Schilling-Vacaflor y Flemmer 2015: 819).

Mientras que se producía una dramática expansión de las actividades extractivas, Fujimori dejó la presidencia, marcando el inicio de la redemocratización en Perú. Hubo entonces una mayor libertad para movilizarse. En un contexto de partidos políticos débilmente institucionalizados, que no canalizan las preferencias de los votantes, las comunidades salieron a las calles para expresar abiertamente sus demandas (Arce 2014). El conflicto socio-ambiental se destacó como particularmente relevante y frecuente en Perú en relación con otros tipos de conflictos sociales. Por ejemplo, los reclamos vinculados a recursos naturales causaron el 41,7 por ciento de todos los conflictos seguidos por la Defensoría del Pueblo entre 2006 y 2011 (Defensoría del Pueblo Adjuntía para la Prevención de Conflictos Sociales y la Gobernabilidad 2012: 38). En algunos casos, los conflictos referían a los impactos ambientales de la minería o de los hidrocarburos, mientras que en otros, las comunidades exigían mayor acceso a los recursos generados por la extracción o simplemente más inversión directa de empresas en el desarrollo local, provincial o regional (De Echave et al. 2009; Arellano Yanguas 2011; Arce 2014; Ponce y McClintock 2014; Gustafsson 2018).

El gobierno nacional respondió a la movilización social con un enfoque reactivo y de arriba hacia abajo. Ordenaba represión policial y realizaba mesas de diálogo ad hoc a fin de buscar soluciones: funcionarios gubernamentales de alto nivel, a menudo ministros, viajaban a zonas extractivas para reunirse con líderes de la sociedad civil. A partir de la década de 2000, la gestión de estos 
conflictos se institucionalizó cada vez más bajo la Presidencia del Consejo de Ministros (Paredes y de la Puente 2017).

La audiencia pública del EIA, una reunión informativa sobre el proyecto que se planea desarrollar, se instituyó en 1996 en los sectores de energía y minería (Solano 1999: 40). El reglamento de diciembre de 2002 trasladó la ubicación de la audiencia de Lima a la zona de extracción para incluir a las comunidades impactadas. Además, el reglamento introdujo talleres locales preliminares para informarle al público del proyecto en cuestión mientras se elaboraba el borrador del EIA. La Dirección General de Asuntos Ambientales del Ministerio de Energía y Minas (MINEM) estaba a cargo de liderar las audiencias públicas y quienes lideraban el proyecto dirigían los talleres. ${ }^{11}$

\section{USOS ESTRATÉGICOS DE LAS AUDIENCIAS PÚBLICAS EN CONFLICTOS EXTRACTIVOS}

Este artículo ayuda a explicar la variación en los usos estratégicos de la audiencia del EIA por parte de las comunidades locales. Para ello, desarrolla un análisis inductivo de trece conflictos, de los cuales cuatro son tratados en detalle.

La autora seleccionó los casos en base a su revisión de fuentes secundarias y a entrevistas realizadas con investigadores de ONGs y académicos especializados en conflictos extractivos durante viajes de trabajo de campo a Lima, Perú, en 2012 y 2017. La muestra incluye los conflictos extractivos emblemáticos ocurridos a partir de fines de 2002, cuando las audiencias del EIA se trasladaron a la zona de extracción, y que tuvieron lugar durante o antes del licenciamiento ambiental. $^{12}$

Resulta importante incluir en la muestra los conflictos emblemáticos que ocurrieron antes de las audiencias para tomar en cuenta la movilización preventiva, es decir, aquella que busca impedir el inicio de un proyecto. La investigación no examina los casos negativos, ya que no busca explicar el conflicto en sí, sino la variación en el papel que juega la audiencia pública en el conflicto. Del mismo modo, la investigación no intenta explicar por qué algunos conflictos ocurrieron antes de la etapa de la audiencia pública, y otros ocurrieron durante esa etapa.

Los datos sobre los casos seleccionados se recopilaron durante el trabajo de campo que la autora realizó en Lima en 2012, 2017 y 2018. Las fuentes incluye-

11 Resolución Ministerial No 596-2002-EM/DM, “Aprueba el Reglamento de Consulta y Participación Ciudadana en el Procedimiento de Aprobación de los Estudios Ambientales en el Sector Energía y Minas"; y entrevista con la autora, Manuel Glave Testino, Investigador Principal de GRADE, por Skype, 10 de agosto de 2018 .

12 Un caso que fue excluido del estudio solo después de investigarlo es el conflicto minero Tintaya/Antapaccay. Este conflicto empezó a crecer de manera notable en la segunda mitad de 2011, más de un año después de la aprobación del EIA del proyecto de expansión Antapaccay de la mina Tintaya. 
ron entrevistas con expertos realizadas por la autora, ${ }^{13}$ artículos de periódicos, documentos gubernamentales y fuentes secundarias.

En los trece conflictos, las comunidades utilizaron las audiencias públicas del EIA de las siguientes formas: (1) participaron activamente en audiencias públicas, (2) bloquearon las audiencias, (3) se quejaron de su exclusión de las audiencias y (4) argumentaron que, en el área afectada por el proyecto extractivo, protecciones de la tierra o la falta de permisos para el uso de la tierra descartaba la posibilidad de un proyecto.

Esta investigación identifica tres vías causales que dan cuenta de las diferentes formas de utilización de las audiencias públicas. Las vías dependen, en primer lugar, de la etapa del proyecto en la que se produce el conflicto: antes del EIA o durante el EIA. Además, importa si la comunidad movilizada en el conflicto está incluida o no en el proceso participativo del EIA, que depende, a su vez, de su localización geográfica respecto del proyecto; y si la comunidad está unida o fragmentada. La figura 1 resume el argumento.

Figura 1: Usos locales de la audiencia pública de EIA en los conflictos peruanos en torno a nuevas extracciones

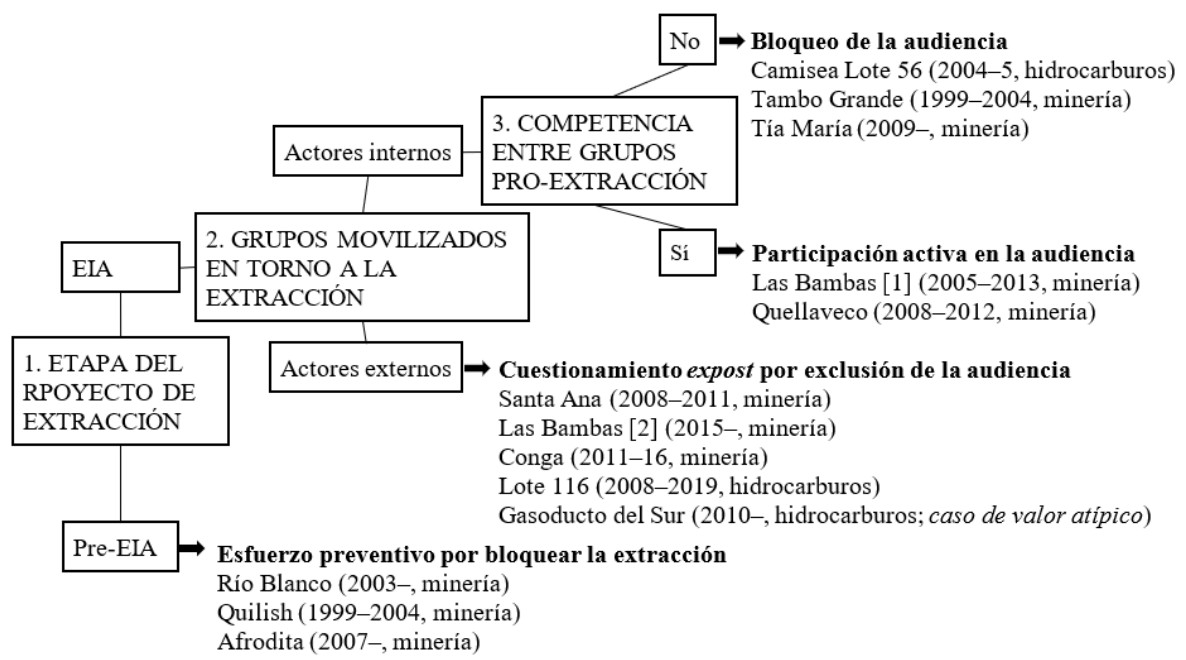

La primera variable es la etapa en que se encuentra el proyecto propuesto al momento que se desata el conflicto. La movilización puede ocurrir antes de que se programe la audiencia pública del EIA. Ahí donde el activismo se desarrolló en ese momento temprano en la muestra de casos, las comunidades buscaron evitar el desarrollo minero y utilizaron varias tácticas. Sin embargo, un factor común en los tres conflictos analizados fue el cuestionamiento al derecho al

13 La autora entrevistó a representantes de ONGs, funcionarios del gobierno, activistas, académicos, consultores, mediadores de conflicto, representantes de compañías mineras, y periodistas. 
uso de la tierra. Las comunidades que se opusieron a las actividades mineras de Afrodita usaron la vía judicial para defender las dimensiones originales de un parque nacional que cubre el territorio en que se ubicaron las concesiones mineras (Ruiz Molleda 2017). Las comunidades apoyaron la creación de una reserva municipal protegida en el sitio de proyecto Quilish (Arce 2014: 92-93). Y las comunidades impactadas por la mina Río Blanco denunciaron la exploración como ilegal con el argumento de que al dueño del proyecto le faltaban los permisos necesarios para usar sus tierras (véase abajo).

Si el conflicto ocurre en la etapa de la audiencia del EIA, las comunidades tienen distintas opciones sobre cómo utilizar la audiencia pública en sus esfuerzos de movilización. En los casos estudiados, las elecciones de las comunidades dependieron en parte de una segunda variable: su relación inicial con la extracción. Para un proyecto, el EIA define el área de influencia $\mathrm{y}$, dentro de ella, el área de influencia directa (O'Faircheallaigh 2017: 1182-84). Solo las comunidades que están en el área de influencia-y a veces en el área de influencia directa-pueden participar en las audiencias (en la figura 1, los "actores internos," a diferencia de los "actores externos").

Las comunidades que no fueron incluidas en el EIA, pero que sí se percibirían como seriamente impactadas por el proyecto analizado, cuestionaron su exclusión. Eso ocurrió en varios casos, como en el conflicto de Santa Ana, discutido más adelante. En el caso Conga, las comunidades argumentaron que el área de influencia era más grande que la definida por el EIA, y que habrían sido incluidas en la audiencia pública si se hubieran reconocido los límites apropiados. Las comunidades adquirieron fuerza con ese argumento, y durante el conflicto, el EIA fue deslegitimado (Jaskoski 2014: 876-77). En la segunda etapa del conflicto Las Bambas ("Las Bambas [2]"), las comunidades se movilizaron para impugnar la ausencia absoluta de un proceso participativo: el estado había estimado que los cambios realizados al diseño del complejo minero habían sido menores. Por lo tanto, el proyecto modificado había avanzado sin participación popular (CooperAcción n.d.; 2015: 2-5, 13; Del Río 2015). En el caso del Lote 116 , las comunidades que se opusieron a un proyecto hidrocarburífero fueron excluidas de la audiencia pública. Estas comunidades se sintieron agraviadas y organizaron su propia asamblea para discutir el EIA (Inforegión 2011; Estrada 2013; CAAAP y CooperAcción 2015: 56-57). El conflicto sobre el Gasoducto del Sur es un caso interesante y atípico. Las comunidades movilizadas en este caso se ubicaban fuera del área de influencia del proyecto $y$, por lo tanto, del proceso participativo del EIA. Sin embargo, no cuestionaron su posición externa ni su exclusión de las audiencias públicas, las cuales no fueron relevantes en el conflicto.

Por último, algunos conflictos involucraron a comunidades internas al área de influencia del proyecto y que se encontraban movilizadas. Los enfoques de los actores internos variaron dependiendo de un tercer y último factor: la competencia con los grupos a favor de la extracción. Algunos activistas internos enfrentaron una fuerte competencia por parte de miembros de su comunidad o de 
otra comunidad interna que apoyaban el desarrollo del proyecto extractivo. Las divisiones en el área de influencia y de influencia directa fueron generadas por las inversiones empresariales en las comunidades. Además, en el caso Quellaveco, la situación estructural también importó: los activistas pertenecían al sector agropecuario, estaban muy organizados (consistente con Arce 2014: 22) y consideraban a la mina como una amenaza.

Ahí donde hubo competencia, participaron grupos de todos los sectores involucrados para asegurar ser escuchados. Esto ocurrió en el conflicto Las Bambas [1] (véase abajo) y, luego de que los activistas fracasaron en impedir la audiencia, en el caso Quellaveco. ${ }^{14}$

Las comunidades que no tuvieron tal división, retrasaron los proyectos negándose a participar en la audiencia pública (Camisea, Lote 56; véase abajo), planificando protestas en las fechas de las audiencias para fomentar boicots (Tambo Grande; véase Arce 2014: 74), o protestando durante la audiencia para detenerla (Tía María; véase Jaskoski 2014: 875-76, y Pinto Herrera 2016: 203, 209).

\section{CUATRO CONFLICTOS}

Los cuatro estudios de caso presentados a continuación utilizan este marco analítico que identifica las vías hacia diversos usos de la audiencia del EIA. El análisis de cada caso-Río Blanco; Santa Ana; Camisea, Lote 56; y Las Bambas [1]—analiza el uso del EIA en el contexto del conflicto más amplio.

\section{Esfuerzo preventivo para bloquear la extracción: Río Blanco y los permisos de tierras}

El proyecto de la mina de cobre a tajo abierto Río Blanco se ubica en la región de Piura al norte de Perú. Ha representado una amenaza para las economías agrícolas de Piura y para el sitio del proyecto, en las comunidades de Yanta y de Segunda y Cajas (A. Bebbington et al. 2007: 13-14; A. Bebbington 2012: 72-73; Gustafsson 2018: 74). El conflicto llegó a su nivel más intenso entre 2003 y 2007, antes de la etapa de la audiencia pública del EIA. Como no pudieron usar la audiencia durante ese período, las dos comunidades, y el movimiento más amplio que surgió en torno al proyecto, emplearon la acción preventiva. Se enfocaron en el derecho al uso de la tierra en el sitio de la exploración: denun-

14 Sobre Quellaveco, véase Ministerio de Energía y Minas Informe No 389-2010/MEM-AAM/CAG/WAL/ CMC, “Evaluación de la Modificación del Estudio de Impacto Ambiental del Proyecto Quellaveco de la empresa Anglo American Quellaveco S.A." (4); Ministerio de Energia y Minas Informe Nº 531-2009/MEMAAM/PRN/WAL/EA/WBF/PRR/RC, “Evaluación de la Modificación del Estudio de Impacto Ambiental del Proyecto Quellaveco de Anglo American Quellaveco S.A." (Anexo I); Coordinadora nacional de radio (2010); y Andina (2010). 
ciaron que la empresa minera carecía de la autorización legítima para utilizar la tierra comunal.

La organización en oposición a Río Blanco comenzó cuando las comunidades de Yanta y de Segunda y Cajas se enteraron de que el dueño de la mina, Minera Majaz S.A.-subsidiaria de la empresa británica Monterrico Metals-planeaba realizar exploraciones en sus tierras. La empresa carecía de la aprobación previa necesaria de las asambleas generales de las comunidades (Defensoría del Pueblo 2006: 4-5, 7-9; véase también A. Bebbington et al. 2007: 21-23). Majaz solicitó la aprobación del estudio ambiental (EA) para la exploración en enero de 2003. Como evidencia de su derecho al uso de la tierra, la empresa presentó acuerdos que había hecho en 2002 con líderes de las dos comunidades, dejando de lado ambas asambleas generales (Defensoría del Pueblo 2006: 7-9; A. Bebbington et al. 2007: 23). También hizo referencia a un permiso de 1997 que Segunda y Cajas le había otorgado al dueño anterior (Defensoría del Pueblo 2006: 6-7, 9). El MINEM aprobó el EA a fines de noviembre de 2003 (De Echave et al 2009: 51).

Segunda y Cajas y Yanta revocaron los supuestos permisos de 2002 en asambleas celebradas en mayo de 2003 y principios de enero de 2004, respectivamente (A. Bebbington et al. 2007: 23). Además, se opusieron abiertamente a la exploración durante los talleres del EIA realizados en Segunda y Cajas en agosto de 2003 y en Yanta en enero de 2004 (De Echave et al. 2009: 51; Gustafsson 2018: 86-87).

Las comunidades mantuvieron su postura respecto de los permisos de uso de la tierra durante las grandes olas de protesta en contra de la mina, que tuvieron lugar en 2004 y 2005. Después de cada episodio de protesta, los esfuerzos oficiales de diálogo fracasaron debido a que las comunidades sostenían que la presencia minera era ilegal. La primera movilización consistió en una marcha de tres días hasta el campamento de la empresa en abril de 2004. En el contexto de esta marcha, la policía mató a un miembro de la comunidad con una granada de gas lacrimógeno. La movilización continuó en los meses siguientes. Un evento visible de esta ola de protesta fue el secuestro de empleados de la empresa por parte de miembros de rondas campesinas, organizaciones comunitarias de autodefensa que también proveían un tipo de estado de derecho (De Echave et al. 2009: 54-55; Gustafsson 2018: 91-92).

En respuesta a la marcha de abril de 2004, el presidente regional de Piura estableció la Mesa de Concertación Río Blanco con el fin de avanzar con el proyecto minero y generar una instancia de participación adicional en el EIA. Los representantes de la comunidad abandonaron la mesa después de unas pocas reuniones, quejándose de la presencia limitada de representantes de la comunidad, la exclusión de las rondas campesinas, y la falta de oportunidades para discutir los impactos ambientales y el tema de la (i)legalidad de los permisos de tierras (A. Bebbington 2012: 75; Gustafsson 2018: 92-93). 
Este patrón de conducta-la protesta y el colapso de diálogo-se vio nuevamente a partir de 2005, cuando 3.000 personas marcharon hacia el campamento minero. Allí se encontraron con la policía nacional y con fuerzas de seguridad privadas. Dos activistas murieron en los enfrentamientos que tuvieron lugar en el campamento, y treinta y dos personas fueron detenidas en los dos días siguientes. El daño físico y psicológico infringido a los detenidos atrajo la atención internacional, involucrando a ONGs europeas que luego ayudarían a presentar una demanda en contra de Monterrico Metals ante el Tribunal Superior de Inglaterra (A. Bebbington 2012: 75-76; Gustafsson 2018: 94).

En respuesta a la violencia, el gobierno central lanzó una instancia de diálogo en 2006. Esta incluyó al Frente por el Desarrollo Sostenible de la Frontera Norte del Perú (FDSFNP), una organización regional que ahora lideraba la oposición a Río Blanco (Gustafsson 2018: 104-107). Sin embargo, la agenda de diálogo omitió el eje principal de la agenda del FDSFNP: los permisos de tierras y la ilegitimidad de la presencia minera. Ante la falta de acuerdo sobre la agenda, El MINEM suspendió el diálogo después de tres reuniones (De Echave et al. 2009: 62-63; Gustafsson 2018: 114-15).

Luego de este segundo intento fallido de diálogo, el FDSFNP, con el apoyo de la Defensoría, se centró en el tema de la falta de permisos para explorar. El MINEM puso fin al asunto a fines de 2006 al determinar que la exploración no requería permiso de la comunidad (Defensoría del Pueblo 2006; A. Bebbington et al. 2007: 32).

A pesar de la decisión de MINEM, el movimiento seguía sosteniendo que la empresa violaba la ley al operar en tierras de las comunidades. En una consulta popular informal en Piura en 2007, el noventa y ocho por ciento de los votos, en tres distritos, fue en contra de la minería (De Echave et al. 2009: 69-71). En este contexto, el Primer Ministro intentó generar una nueva instancia de diálogo entre las partes. Este esfuerzo fracasó, ya que el FDSFNP se mantuvo firme en que la empresa carecía de derechos de uso de la tierra, mientras que el MINEM se centró en discutir los detalles del desarrollo del proyecto (Gustafsson 2018: 120-21). Después de la consulta popular, el proyecto fue transferido a otra empresa. Río Blanco quedó paralizado: a fines de 2020, la empresa todavía no había entregado el EIA al MINEM (Observatorio de conflictos mineros en Perú 2020: 13-14).

\section{Impugnación a la exclusión de la audiencia del EIA: Santa Ana}

El caso de la mina de plata a tajo abierto Santa Ana, en la región de Puno, ilustra la dinámica por la cual las comunidades movilizadas impugnan su exclusión de la audiencia pública del EIA. La organización en contra de la mina empezó en las comunidades localizadas en el área de influencia indirecta del proyecto. Si bien estas comunidades se percibían como fuertemente impactadas, no fueron incluidas en la etapa participativa del EIA. El conflicto alcanzó su punto 
máximo después de la audiencia pública, cuando activistas de estas comunidades se unieron a otros grupos en el "Aymarazo." Esa movilización afectó el sur de Puno, poblado por la nación indígena aymara, así como la parte norte de la región, principalmente quechua (Quiñones 2013: 18, 22).

La movilización en contra de la exclusión del proceso participativo ambiental de Santa Ana tuvo lugar en un contexto de divisiones entre las comunidades del área de influencia directa, por un lado, y las del área de influencia indirecta, por el otro. Esa división, causada por la mina, comenzó durante la titulación para el proyecto cuando el titular prestó atención especialmente a las comunidades en el área de influencia directa. El proyecto minero pertenecía a Bear Creek Mining Company Sucursal del Perú de Bear Creek Mining Company de Canadá. El área de influencia directa se ubicaba principalmente en el distrito de Huacullani, en la provincia de Chucuito (Quiñones 2013: 25-26, 29). En un comienzo, las concesiones mineras se superponían con la reserva protegida Aymara Lupaca en la que la minería estaba prohibida. Jenny Villavicencio, titular de Santa Ana y, a partir de 2006, representante legal de Bear Creek, consiguió el apoyo de autoridades comunales de Huacullani para modificar los límites de Aymara Lupaca (Quiñones 2013: 35, 37-39, 45-46). Un decreto ejecutivo de enero de 2006 redujo la extensión de la reserva, dejando a Santa Ana fuera de ella. En 2006 y 2007, se otorgaron las concesiones mineras a Villavicencio (Quiñones 2013: 41-42). En 2007, el Consejo de Ministros le dio a Villavicencio un permiso especial para obtener las concesiones. Esta autorización fue necesaria debido a la existencia de restricciones constitucionales a las inversiones mineras extranjeras en zonas fronterizas (Decreto Supremo N ${ }^{\circ}$ 083-2007-EM, citado por Quiñones 2013; 42-43).

La primera movilización masiva contra Santa Ana tuvo lugar en 2008, año en que comenzó la exploración sostenida del proyecto. Las actividades exploratorias alertaron a las comunidades de Chucuito de la existencia del proyecto y generaron preocupación en la provincia sobre los impactos ambientales de la mina (Quiñones 2013: 60).

Ya para 2008 había fuertes divisiones en el área de influencia de la mina. Varios líderes locales desconocían el proceso de transferencia de títulos y lo resintieron. Además, debido a que las inversiones de la compañía en programas socioeconómicos se limitaban al área de influencia directa, gran parte de Huacullani apoyaba la mina, mientras que los distritos vecinos, así como la comunidad de Yorocco-ubicada en Huacullani pero fuera del área de influencia directaeran críticos de la misma. En medio de estos desacuerdos, la movilización en contra de Santa Ana tuvo lugar fuera del área de influencia directa (Quiñones 2013: 40, 47-51, 59-61).

En octubre de 2008, una protesta culminó con la toma del campamento minero y la destrucción de las oficinas de la empresa. Esta protesta fue liderada por el Frente de Defensa de los Recursos Naturales de la Zona Sur de Puno (FDRN- 
ZS), formado para oponerse al proyecto de Santa Ana. La protesta resultó en la interrupción de la exploración (Pinto Herrera 2013: 214; Quiñones 2013: 50, 61).

Después de los acontecimientos de 2008, el proceso de revisión del EIA intensificó las divisiones entre las comunidades afectadas directa e indirectamente por el proyecto y fue fundamental para desencadenar el Aymarazo (Pinto Herrera 2013: 213; Quiñones 2013: 61-62, 64-65). En este período las comunidades excluidas de los talleres y la audiencia pública del EIA se movilizaron, criticaron su exclusión, y se reunieron para discutir el proyecto de mina por fuera de la estructura participativa formal.

Sólo las comunidades ubicadas en el área de influencia directa de la mina fueron incluidas en los talleres y la audiencia del EIA. Aquellos movilizados por fuera de la estructura participativa se quejaron de que los cuarentaiocho talleres del EIA fueron realizados (en 2009) en el área de influencia directa. El FDRNZS dirigió sus propias reuniones que fueron críticas respecto de la mina (Pinto Herrera 2013: 211-12; Quiñones 2013: 52-57). A fines de febrero de 2011, aproximadamente 1.000 simpatizantes de Santa Ana asistieron a la audiencia de EIA, que se desarrolló sin incidentes. Posteriormente, las comunidades ubicadas fuera del área de influencia directa expresaron su malestar por haber sido excluidas del proceso (Hualpa 2011; Pinto Herrera 2013: 213; Uceda 2014).

La movilización en contra de Santa Ana se intensificó a partir de marzo de 2011. En respuesta a los reclamos de las organizaciones de la sociedad civil, incluido el FDRNZS, el consejo regional de Puno emitió una ordenanza regional que prohibió la minería en toda la región. (Esta medida fue simbólica ya que el gobierno central era responsable de la emisión de concesiones mineras; Hualpa 2011; Cavero 2014: 26-27). Cuando el presidente de Puno se negó a firmar la ordenanza, 2.000 personas marcharon hacia la ciudad de Puno, el 30 de marzo. A fines de abril, una huelga de cuarentaiocho horas contra el gobierno regional y la mina paralizó el sur de Puno (Cavero 2014: 70-71; Defensoría del Pueblo Adjuntía para la Prevención de Conflictos Sociales y Gobernabilidad 2016: 4).

En mayo de 2011 comenzó otra ola de huelgas en torno al conflicto minero. La movilización se extendía por Puno. En el Aymarazo, más de 4.000 personas bloquearon las principales carreteras que conectan las regiones de Puno y Tacna, y Perú y Bolivia. El conflicto se hizo más intenso el 24 de junio de 2011, cuando personal policial y soldados del ejército dispararon contra los manifestantes que intentaban tomar control del aeropuerto regional, matando a seis activistas (Pinto Herrera 2013: 214-17; Defensoría del Pueblo Adjuntía para la Prevención de Conflictos Sociales y Gobernabilidad 2016: 5).

La movilización se expandió no solo geográficamente, sino que también abarcó más temas. Varios grupos se sumaron a la movilización para frenar otros proyectos de gran escala en el sur de Puno, exigir una ley de consulta previa, o con el fin de oponerse a la minería informal por sus impactos ambientales adversos (Pinto Herrera 2013: 210, 215-17; Quiñones 2013: 17). 
El Aymarazo terminó a fines de junio luego de que el gobierno revocara el permiso para la actividad minera cerca de la frontera y suspendiera la emisión de los nuevos permisos mineros en toda la región por un período de tres años (Pinto Herrera 2013: 217-18; Quiñones 2013: 18-19).

\section{Los actores internos unidos bloquean la audiencia: Camisea, Lote 56}

Distinto al conflicto por Santa Ana, las comunidades que se movilizaron en el caso del Lote 56 de gas natural de Camisea eran actores internos-o sea, grupos que habían sido incluidos en la etapa participativa del EIA. Además, estas comunidades se encontraban unidas, sin conflictos internos que las dividieran. Como una ilustración de la tercera vía en el modelo causal de esta investigación, las organizaciones representativas de las comunidades impactadas se negaron a participar en la audiencia y, de esa manera, impidieron que se celebrara. Al final, la audiencia avanzó debido a la presión empresarial sobre las organizaciones.

Como contexto, los campos de Camisea en la región de Cusco abarcan un área que incluye la cuenca del Bajo Urubamba y que está poblada por comunidades indígenas, principalmente de la nación Machiguenga (Ross 2008: 203-204). En el momento en que se revisaba el proyecto del Lote 56, la explotación del otro campo de Camisea, el Lote 88, ya había transformado la realidad social y económica local, tal que ahora los Machiguenga dependían de la industria del gas (Earle y Pratt 2009: 18; Bruijn y Whiteman 2010).

Dos organizaciones indígenas locales: el Consejo Machiguenga del Río Urubamba (COMARU), que representaba a treinta comunidades, y la Central de Comunidades Nativas Machiguengas (CECONAMA), que competía con COMARU y representaba a ocho comunidades (Ross 2008: 216-17), participaron en el conflicto en torno al Lote 56.

La audiencia pública para el Lote 56, fijada originalmente para diciembre de 2004, tendría lugar en la comunidad de Shivankoreni. Las comunidades machiguengas sin embargo exigieron que la audiencia fuera pospuesta porque no se les había proporcionado los noventa días requeridos para revisar el EIA. En respuesta a sus reclamos, la audiencia fue reprogramada para enero de 2005 (Earle y Pratt 2009: 57-58). Sin embargo, antes de la reunión, los Machiguenga se vieron afectados por la rotura de una tubería de gas y por la inacción estatal ante el problema. Por ello, las comunidades decidieron retrasar la audiencia (Ross 2008: 261).

La rotura de la tubería en diciembre de 2004 produjo un importante derrame de gas. La alarma de los Machiguenga por el incidente fue tan intensa que, en enero de 2005, COMARU y CECONAMA presentaron una carta conjunta al MINEM. La carta exigía, entre otras cosas, una explicación sobre las causas de la rotura, una inspección completa del gasoducto y una auditoría indepen- 
diente de todas las actividades del Lote 88 y del gasoducto. En la carta, las organizaciones también sostenían que hasta que no se abordaran sus reclamos, rechazarían cualquier actividad en el Lote 56, incluso amenazaron con impedir la audiencia del EIA (Ross 2008: 249; Earle y Pratt 2009: 57-58).

Las comunidades, con COMARU a la cabeza, impidieron que se llevara a cabo la audiencia de enero mediante un boicot acompañado de fuertes protestas frente al sitio de reunión. La audiencia fue reprogramada nuevamente para el 8 de mayo de 2005. Ya que el MINEM no había respondido a la carta de enero de COMARU y CECONAMA, la dirigencia de Shivankoreni notificó a las empresas y al MINEM que las condiciones existentes no eran las adecuadas para la reunión (Griffiths 2007: 13; Ross 2008: 251).

Las presiones de las compañías finalmente dividieron a los Machiguenga y aseguraron que la audiencia se llevase a cabo. El MINEM y los funcionarios de las empresas de Camisea llegaron a Shivankoreni el 8 de mayo. La comunidad estaba vacía porque los residentes se encontraban participando en un día de trabajo programado en la parcela de tierra comunal (la chacra), a una cierta distancia. Los empresarios luego invitaron a los jefes de COMARU y CECONAMA a una reunión en su sede local, donde los persuadieron para que apoyaran la audiencia del EIA. El líder de COMARU advirtió a los líderes de Shivankoreni que la compensación por el proyecto podría depender de la cooperación y, de esa manera, los convenció para que permitieran la audiencia. Esta fue celebrada el 9 de mayo de 2005, aunque no asistieron todas las comunidades. El MINEM aprobó el EIA en julio de 2005 (Griffiths 2007: 13-14; Ross 2008: 250-51; Earle y Pratt 2009: 58-60) y la producción en el Lote 56 comenzó en 2008 (Suárez 2008).

\section{Participación activa por parte de actores internos divididos: Las Bambas [1]}

El cuarto y último conflicto fue por la enorme mina Las Bambas en la provincia de Cotabambas, en la región sur de Apurímac. La movilización social en este caso surgió en las comunidades del área de influencia de la mina que, como en el caso Camisea, fueron incluidas en la etapa participativa del EIA. Sin embargo, a diferencia del caso machiguenga, hubo fuertes divisiones entre las comunidades impactadas por Las Bambas antes de y durante el proceso participativo. Las divisiones se relacionaron con la cantidad de apoyo social que las comunidades recibían por la mina, que era marcadamente dispar. Representantes de todas las comunidades "internas" participaron en la audiencia del EIA para expresar sus intereses específicos.

Las divisiones en el área de influencia de Las Bambas en gran parte emergieron como consecuencia de los recursos excepcionales otorgados a una sola comunidad, Fuerabamba, que era la única comunidad cuyo reasentamiento estaba programado. Este favoritismo fue evidente en los proyectos sociales coordina- 
dos por Proinversión, la agencia estatal que promovía la inversión privada en importantes proyectos gubernamentales. Después de la transferencia del proyecto del estado a Xstrata AG Suiza en 2004, el nuevo dueño también orientó sus inversiones hacia Fuerabamba (Gustafsson 2018: 144-46, 149, 167-168).

La primera gran protesta de los actores internos en el conflicto de Las Bambas consistió en una huelga de cuarentaiocho horas en Apurímac en marzo de 2005, seguida de huelgas de gran escala en febrero de 2006 y julio de 2007. En las tres movilizaciones, comunidades de Cotabambas se encaminaron a conseguir el control de un fondo social de \$64 millones que el gobierno había creado cuando se transfirió el proyecto a Xstrata. Los manifestantes sostenían que Proinversión administraba mal el fideicomiso, al descuidar la agricultura e implementar proyectos con lentitud. De manera más general, las comunidades percibían que la distribución de fondos por la provincia no era adecuada (CooperAcción 2015: 14-15; Gustafsson 2018: 143, 146-151). ${ }^{15}$

Después de la gran movilización de 2007, las negociaciones entre los activistas y el MINEM resultaron en la reconstitución de la gerencia del fideicomiso, que estaría conformada por alcaldes y representantes de Xstrata. A principios de 2009, el fideicomiso se convirtió en el Fondo Social Las Bambas (FOSBAM), y formó parte de un cambio legal nacional orientado a poner fin al control del gobierno central sobre la inversión social de las empresas privadas en los sectores extractivos. De acuerdo con dicho cambio, las áreas directamente afectadas recibirían el 85 por ciento de la inversión de FOSBAM (CooperAcción 2015: 15; Gustafsson 2018: 151-53).

Otra gran protesta en el conflicto de Las Bambas fue una huelga de dos días en mayo de 2011. Esta protesta evidenció la importancia de la compensación y los beneficios materiales además de la capacidad de las empresas extractivas de dividir las comunidades. Las catorce comunidades que participaron en la huelga fueron clasificadas como mínimamente impactadas por la mina y por lo tanto recibían poco apoyo (Gustafsson 2018: 180-82). En la huelga, un ingeniero de la empresa fue secuestrado, y la policía nacional reprimió violentamente a los manifestantes (CooperAcción 2015: 16). Para resolver el conflicto, Xstrata prometió inversiones en desarrollo social y puestos de trabajo (Defensoría del Pueblo, Adjuntía para la Prevención de Conflictos Sociales y la Gobernabilidad 2017: 3-4; Gustafsson 2018: 182).

Con respecto a la participación en el EIA en el caso Las Bambas, cada una de las comunidades impactadas expresó sus demandas materiales durante el desarrollo del EIA, en el seno de las reuniones formales. Para la etapa participativa del EIA, las comunidades del área de influencia directa se encontraban muy desarticuladas debido a las negociaciones mantenidas de forma individual entre Xstrata y las comunidades (Gustafsson 2018: 178-89, 181). Los talleres del

15 Como la lucha por un rol en el fideicomiso fue con el estado y no con Xstrata con que Fuerabamba negociaba, esa comunidad la apoyó (Gustafsson 2018: 149, 170-71). 
EIA se llevaron a cabo durante 2009 y 2010. En las reuniones, las autoridades políticas locales, las organizaciones de la sociedad civil, y la población expresaron preocupaciones ambientales y socioeconómicas (Moreno Hermoza 2014: 138-140; CooperAcción 2015: 3). Todas las comunidades del área de influencia de Las Bambas asistieron a la audiencia del EIA de julio de 2010, en la que participaron aproximadamente 4.500 personas. El evento se desarrolló sin mayores contratiempos e implicó un intercambio abierto de ideas. El MINEM aprobó el EIA en marzo de 2011 (Moreno Hermoza 2014: 139; CooperAcción 2015: 6).

\section{CONCLUSIÓN}

Este análisis de los principales conflictos extractivos en Perú identifica y explica la variación respecto de cómo las comunidades locales organizadas han utilizado la audiencia del EIA en dichos conflictos.

La explicación se centra en tres factores claves. El primero consiste en la etapa del proyecto extractivo en que sucede el conflicto. El caso de Río Blanco, por ejemplo, ilustra la estrategia de las comunidades en un conflicto que ocurre antes de la programación de la audiencia del EIA. Estas comunidades bloquearon la extracción al centrar la discusión en la situación legal del predio donde se desarrollaba el proyecto minero. Dos comunidades-y, eventualmente, un movimiento regional-insistieron en que la mera presencia de la empresa era ilegal debido a que ésta carecía de permisos de uso de la tierra.

Un segundo factor importante consiste en si, para los propósitos del proceso participativo del EIA, el diseño del proyecto coloca a los actores movilizados dentro o fuera del área de influencia o de influencia directa del proyecto. Como vimos en el caso de Santa Ana, las comunidades "externas," que no son invitadas a la audiencia de EIA, pueden movilizarse en reacción a esa exclusión. Las comunidades que se encontraban fuera del área de influencia directa de la mina cuestionaron su exclusión y participaron en reuniones informales ante la falta de acceso a las formales. La exclusión contribuyó a ampliar la movilización social, que culminó en el Aymarazo.

Finalmente, el tercer factor depende de los actores "internos," y consiste en su nivel de cohesión social. Una comunidad interna unida puede impedir la celebración de la audiencia pública. Ese empleo de la audiencia se ve en el caso Camisea. En otros conflictos, por el contrario, los actores internos no están de acuerdo sobre la extracción. En estos casos, las comunidades-con sus distintos pedidos-se quedan con una única opción: la de participar activamente en la audiencia pública a fin de ser escuchadas. Este último empleo de la audiencia se observa en Las Bambas.

El estudio de los usos estratégicos de la audiencia del EIA por parte de las comunidades afectadas tiene implicancias para el modo en que pensamos las instituciones participativas y el conflicto extractivo en general. Este trabajo con- 
tribuye a nuestra comprensión de la relación entre la participación y el conflicto al enfocarse no sólo en la participación en un espacio participativo establecido, sino también en cómo las comunidades se organizan alrededor de estas instituciones. En última instancia, el artículo demuestra la relevancia inesperada de una institución que formalmente es un vehículo limitado para la participación. Mientras que las audiencias del EIA son, de acuerdo con los reglamentos, puramente informativas, las comunidades organizadas pueden usarlas estratégicamente en conflictos importantes con el fin de detener proyectos extractivos de gran escala. Es decir, la institución participativa de la audiencia pública, que durante "tiempos normales" quizás sirva como herramienta del estado y de las empresas para cooptar, debilitar y controlar comunidades, puede volverse un importante activo para las comunidades que se movilizan frente a la extracción.

\section{REFERENCIAS}

Andina. 2010, 16 de abril. "Hasta el 9 de mayo se recibirán aportes al EIA del proyecto minero de Quellaveco." Recuperado el 27 de junio de 2013 de http:/ /www.andina.com.pe/ ingles/Noticia.aspx?id=Kah5223XWD0=Andina.

Arce, Moisés. 2014. Resource Extraction and Protest in Peru. Pittsburgh: University of Pittsburgh Press.

Arellano Yanguas, Javier. 2011. ¿Minería sin fronteras? Conflicto y desarrollo en regiones mineras del Perú. Lima: Instituto de Estudios Peruanos.

Barandiarán, Javiera, y Sebastián Rubiano-Galvis. 2019. “An Empirical Study of EIA Litigation Involving Energy Facilities in Chile and Colombia." Environmental Impact Assessment Review 79 (noviembre): https:/ /doi.org/10.1016/j.eiar.2019.106311.

Bebbington, Anthony. 2010. "Extractive Industries and Stunted States: Conflict, Responsibility and Institutional Change in the Andes." En Corporate Social Responsibility: Comparative Critiques, editado por K. Ravi Raman y Ronnie D. Lipschutz. New York: Palgrave Macmillan, 97-115.

Bebbington, Anthony. 2012. "Social conflict and Emergent Institutions: Hypotheses from Piura, Peru." En Social Conflict, Economic Development and Extractive Industry: Evidence from South America, editado por Anthony Bebbington. London: Routledge, 67-88.

Bebbington, Anthony, y Jeffrey Bury (eds.). 2013. Subterranean Struggles: New Dynamics of Mining, Oil, and Gas in Latin America. Austin: University of Texas Press.

Bebbington, Anthony, Michael Connarty, Wendy Coxshall, Hugh O'Shaughnessy, y Mark Williams. 2007. Minería y desarrollo en el Perú con especial referencia al Proyecto Río Blanco, Piura. Lima: Oxfam Internacional, Instituto de Estudios Peruanos, Centro de Investigación y Promoción del Campesino, Peru Support Group.

Bebbington, Anthony, Denise H. Bebbington, y Jeffrey Bury. 2010. "Federating and Defending: Water, Territory and Extraction in the Andes." En Out of the Mainstream: Water Rights, Politics and Identity, editado por Rutgerd Boelens, David Getches, y Armando Guevara-Gil. London: Earthscan, 307-327.

Bebbington, Denise Humphreys. 2012. "Consultation, Compensation and Conflict: Natural Gas Extraction in Weenhayek Territory, Bolivia." Journal of Latin American Geography 11(2): 49-71.

Bruijn, Eveline, y Gail Whiteman. 2010. “That Which Doesn't Break Us: Identity Work by Local Indigenous 'Stakeholders.'” Journal of Business Ethics 96 (octubre): 479-495.

Bury, Jeffrey, y Timothy Norris. 2013. "Rocks, Rangers, and Resistance: Mining and Conservation Frontiers in the Cordillera Huayhuash, Peru." En Subterranean Struggles: New Dynamics of Mining, Oil, and Gas in Latin America, editado por Anthony Bebbington y Jeffrey Bury. Austin: University of Texas Press, 91-118. 
Carcelén Pacheco, Jerónimo, y Valentina Mir Bennett. 2014. "Case Study: Chile." Americas Quarterly 8(2): 62-67.

Cavero, Omar. 2014. Hacia un entendimiento de la conflictividad social: Las protestas contra la minería en Puno. Cuaderno de trabajo No. 21 (diciembre). Lima: Pontificia Universidad Católica del Perú, Departamento de Ciencias Sociales.

Centro Amazónico de Antropología y Aplicación Práctica (CAAAP) y CooperAcción. 2015. Estudio sobre la actuación de las empresas petroleras Perenco en el Lote 67 y Maurel Et Prom-Pacific Rubiales Energy en el Lote 116: Impactos socio-ambientales y afectación de derechos de los pueblos indígenas Awajun y Wampis en Amazonas y Kechwa y Arabela en Loreto. Informe Final (abril). Lima: CAAAP y CooperAcción.

CooperAcción. n.d. "Las Bambas: Un proyecto que no para de cambiar-Cinco modificaciones en 20 meses." Folleto. Lima: CooperAcción.

CooperAcción. 2015. Caso "Las Bambas". Informe Especial. Lima: CooperAcción.

CooperAcción. 2018. "Sección económica." Actualidad minera del Perú 228 (junio): 4-7.

Coordinadora Nacional de Radio. 2010, 9 de abril 2010. "Controversia por audiencia sobre proyecto minero Quellaveco." Recuperado el 27 de junio de 2013 de http:/ / www.cnr. org.pe/nota.shtml? $\mathrm{x}=3155$.

De Echave, José, Alejandro Diez, Ludwig Huber, Bruno Revesz, Xavier Ricard Lanata, y Martín Tanaka. 2009. Minería y conflicto social. Lima: Instituto de Estudios Peruanos, Centro de Investigación y Promoción del Campesinado, Centro de Estudios Regionales Andinos Bartolomé de Las Casas, y Consorcio de Investigación Económica y Social.

Defensoría del Pueblo. 2006. Informe No. 001-2006/ASPMA-MA. Lima: Defensoría del Pueblo.

Defensoría del Pueblo Adjuntía para la Prevención de Conflictos Sociales y la Gobernabilidad. 2012. Violencia en los conflictos sociales. Informe Defensorial No. 156 (marzo). Lima: Defensoría del Perú.

Defensoría del Pueblo Adjuntía para la Prevención de Conflictos Sociales y la Gobernabilidad. 2016. "Caso: Santa Ana-Huacullani." Ayuda memoria. RCS No. 57-noviembre 2008. Actualizada al RCS No. 143-enero 2016. Lima: Defensoría del Pueblo.

Defensoría del Pueblo Adjuntía para la Prevención de Conflictos Sociales y la Gobernabilidad. 2017. "Caso: Cotabambas-MMG proyecto minero Las Bambas." Ayuda memoria. RCS No. 87-mayo 2011. Actualizada al RCS No. 157-marzo 2017. Lima: Defensoría del Pueblo.

Delamaza, Gonzalo. 2010. “Conflicto político y diseños institucionales de participación en el caso chileno." Revista de sociología 23: 11-37.

Delamaza, Gonzalo, Antoine Maillet, y Christian Martínez Neira. 2017. "Socio-Territorial Conflicts in Chile: Configuration and Politicization (2005-2014)." European Review of Latin American and Caribbean Studies 104 (julio-diciembre): 23-46.

Del Río, Maria Luisa. 2015, 15 de noviembre. “Las Bambas: Las dos caras del conflicto por el proyecto minero." Perú 21. Recuperado el 11 de mayo de 2017 de http://peru21.pe/ politica/proyecto-minero-bambas-dos-caras-conflicto-fotos-2232126.

Devlin, John F., y Nonita T. Yap. 2008. "Contentious Politics in Environmental Assessment: Blocked Projects and Winning Coalitions." Impact Assessment and Project Appraisal 26(1): 17-27.

Earle, Lucy, y Brian Pratt. 2009. Indigenous Social Movements and International NGOs in the Peruvian Amazon. Occasional Papers Series. No. 49 (marzo). Oxford: International NGO Training and Research Centre.

Eisenstadt, Todd A., y Karleen Jones West. 2019. Who Speaks for Nature? Indigenous Movements, Public Opinion, and the Petro-State in Ecuador. New York: Oxford University Press.

Elías, Silvel, y Geisselle Sánchez. 2014. "Case Study: Guatemala." Americas Quarterly 8(2): $70-75$.

Estrada, Edy. 2013, 25 de abril. "Pronunciamiento de ORPIANP sobre territorio awajún-wampís." Baguapero.com. Recuperado el 2 de marzo de 2018 de http:/ / www.baguaperu. com/2013/04/pronunciamiento-de-orpianp-sobre.html. 
Falleti, Tulia G., y Thea N. Riofrancos. 2018. “Endogenous Participation: Strengthening Prior Consultation in Extractive Economies." World Politics 70(1): 86-121.

Flemmer, Riccarda, y Almut Schilling-Vacaflor. 2016. "Unfulfilled Promises of the Consultation Approach: The Limits to Effective Indigenous Participation in Bolivia's and Peru's Extractive Industries." Third World Quarterly 37(1): 172-188.

Griffiths, Tom. 2007. Holding the IDB and IFC to Account on Camisea II: A Review of Applicable International Standards, Due Diligence and Compliance Issues. Septiembre. San Francisco: Amazon Watch.

Gustafsson, Maria-Therese. 2018. Private Politics and Peasant Mobilization: Mining in Peru. Palgrave MacMillan.

Henao, Juan Carlos, y Ana Carolina González Espinosa (eds). 2016. Minería y comunidades: Impactos, conflictos y participación ciudadana. Minería y desarrollo, Tomo IV. Bogotá: Universidad Externado de Colombia.

Hochstetler, Kathryn. 2011. "The Politics of Environmental Licensing: Energy Projects of the Past and Future in Brazil." Studies in Comparative International Development 46(4): 349-371.

Hualpa, Eliana. 2011, 23 de marzo. “Tensión en Huacullani por proyecto minero Santa Ana." Noticias SER. Recuperado el 8 de marzo de 2018 de http:/ / www.noticiasser. pe/23/03/2011/puno/tension-en-huacullani-por-proyecto-minero-santa-ana.

Inforegión. 2011, 17 de marzo. "Petrolera presenta estudio de impacto ambiental para exploración de pozos en Amazonas." Recuperado el 1 de marzo de 2018 de http:/ /www. inforegion.pe/92523/petrolera-maurel-presenta-estudio-de-impacto-ambiental-para-exploracion-de-pozos-en-amazonas/.

Jaskoski, Maiah. 2014. "Environmental Licensing and Conflict in Peru's Mining Sector: A Path-Dependent Analysis." World Development 64 (diciembre): 873-883.

Jaskoski, Maiah. 2020. "Participatory Institutions as a Focal Point for Mobilizing: Prior Consultation and Indigenous Conflict in Colombia's Extractive Industries." Comparative Politics 52, no. 4 (julio): 537-556.

Leifsen, Esben, Luis Sánchez-Vázquez, y Maleny Gabriela Reyes. 2017. “Claiming Prior Consultation, Monitoring Environmental Impact: Counterwork by the Use of Formal Instruments of Participatory Governance in Ecuador's Emerging Mining Sector." Third World Quarterly 38(5): 1092-1109.

Li, Fabiana. 2015. Unearthing Conflict: Corporate Mining, Activism, and Expertise in Peru. Durham: Duke University Press.

McAdam, Doug, Hilary Schaffer Boudet, Jennifer Davis, Ryan J. Orr, W. Richard Scott, y Raymond E. Levitt. 2010. "'Site Fights': Explaining Opposition to Pipeline Projects in the Developing World." Sociological Forum 25(3): 401-427.

Moreno Hermoza, Gustavo. 2014. "El caso Las Bambas." En Minería, conflicto social y diálogo, editado por Iván Ormachea Choque, Javier Caravedo Chocano, Gustavo Moreno Hermoza, y César Bedoya García. Lima: ProDiálogo, Prevención y Resolución de Conflictos, 125-161.

Observatorio de conflictos mineros en el Perú. 2020. 27o Observatorio de conflictos mineros en el Perú. Reporte segundo semestre (diciembre). Lima: Observatorio de Conflictos Mineros en el Perú, CooperAcción, Fedepaz, Grufides.

O'Faircheallaigh, Ciaran. 2017. "Shaping Projects, Shaping Impacts: Community-Controlled Impact Assessments and Negotiated Agreements." Third World Quarterly 38(5): 1181-1197.

Orduz Salinas, Natalia. 2014. La consulta previa en Colombia. Documento de trabajo ICSO No. 3. Santiago: Instituto de Investigación en Ciencias Sociales, Universidad Diego Portales.

Paredes, Maritza, y Lorena de la Puente. 2017. "The Social Construction of a Public Problem: The Role of the Ombudsman in Building Institutions for Extractive Conflict. En Resource Booms and Institutional Pathways: The Case of the Extractive Industry in Peru, editado por Eduardo Dargent, José Carlos Orihuela, Maritza Paredes, y María Eugenia Ulfe. Palgrave Macmillan, 119-151. 
Pellegrini, Lorenzo, y Murat Arsel. 2018. "Oil and Conflict in the Ecuadorian Amazon: An Exploration of Motives and Objectives." European Review of Latin American and Caribbean Studies 106(julio-diciembre): 209-218.

Pellegrini, Lorenzo, y Marco Octavio Ribera Arismendi. 2012. “Consultation, Compensation and Extraction in Bolivia after the 'Left Turn': The Case of Oil Exploration in the North of La Paz Department." Journal of Latin American Geography 11(2): 103-120.

Pinto Herrera, Honorio. 2013. "Conflicto minero en Santa Ana (Puno)." Investigaciones sociales 17(31): 207-220.

Pinto Herrera, Honorio. 2016. "Proyecto minero Tía María: Razones de la protesta." Investigaciones sociales 20(36): 199-213.

Ponce, Aldo F., y Cynthia McClintock. 2014. “The Explosive Combination of Inefficient Local Bureaucracies and Mining Production: Evidence from Localized Societal Protests in Peru." Latin American Politics and Society 56(3): 118-140.

Quiñones, Patricia. 2013. "Concesiones, participación y conflicto en Puno. El caso del proyecto minero Santa Ana." En Los límites de la expansión minera en el Perú, editado por Javier Torres Seone. Lima: Asociación Servicios Educativos Rurales, 17-71.

Riofrancos, Thea N. 2017. "Scaling Democracy: Participation and Resource Extraction in Latin America." Perspectives on Politics 15(3): 678-696.

Rodríguez-Garavito, César. 2011. "Ethnicity.gov: Global Governance, Indigenous Peoples, and the Right to Prior Consultation in Social Minefields." Indiana Journal of Global Legal Studies 18(1): 263-305.

Ross, Catherine. 2008. "El caso del Proyecto Camisea: Logros y limitaciones de una coalición ciudadana naciente." En Defendiendo derechos y promoviendo cambios: El estado, las empresas extractivas y las comunidades locales en el Perú, editado por Martin Scurrah. Lima: Instituto de Estudios Peruanos y Oxfam América, 199-268.

Ruiz Molleda, Juan Carlos. 2017, 6 de junio. "Caso Ichigkat Muja: Admiten demanda por incumplimiento estatal." Servindi. Recuperado el 26 de abril de 2021 de http: / / www. servindi.org/actualidad-noticias/06/06/2017/admiten-demanda-contra-incumplimiento-del-estado-del-primer-proceso.

Sanborn, Cynthia A., Verónica Hurtado, y Tania Ramírez. 2016. La consulta previa en el Perú: Avances y retos. Documento de investigación 6. Lima: Universidad del Pacífico.

Schilling-Vacaflor, Almut. 2013. "Prior Consultations in Plurinational Bolivia: Democracy, Rights and Real Life Experiences." Latin American and Caribbean Ethnic Studies 8(2): 202-220.

Schilling-Vacaflor, Almut. 2017. "Who Controls the Territory and the Resources? Free, Prior and Informed Consent (FPIC) as a Contested Human Rights Practice in Bolivia." Third World Quarterly 38(5): 1058-1074.

Schilling-Vacaflor, Almut, y Riccarda Flemmer. 2015. "Conflict Transformation through Prior Consultation? Lessons from Peru." Journal of Latin American Studies 47(4): 811-839.

Scurrah, Martin (ed.). 2008. Defendiendo derechos y promoviendo cambios: El estado, las empresas extractivas y las comunidades locales en el Perú. Lima: Instituto de Estudios Peruanos y Oxfam América.

Solano, Pedro. 1999. Hidrocarburos y áreas naturales protegidas: Más allá del suelo y el subsuelo. Lima: Sociedad Peruana de Derecho Ambiental.

Suárez, Cándice. 2008, 13 de octubre. "Consorcio Camisea inició producción de hidrocarburos de Lote 56 con inversión de US\$ 872 millones (ampliación)." Andina. Recuperado el 25 de junio de 2020 de https://andina.pe/agencia/noticia-consorcio-camisea-inicio-produccion-hidrocarburos-lote-56-inversion-872-millones-ampliacion-198553. aspx.

Thorp, Rosemary, Stefania Battistelli, Yvan Guichaoua, José Carlos Orihuela, y Maritza Paredes. 2014. Los desafíos de la minería y el petróleo para el desarrollo: Lecciones de África y Latinoamérica. Lima: Fondo Editorial de la Pontificia Universidad Católica del Perú.

Uceda, Ricardo. 2014, 7 de enero. "Santa Ana: la decisión del mes." La República. Recuperado el 8 de marzo de 2018 de http://larepublica.pe/politica/763490-santa-ana-la-decision-del-mes. 
Walter, Mariana, y Joan Martinez-Alier. 2010. "How to be Heard When Nobody Wants to Listen: Community Action against Mining in Argentina." Canadian Journal of Development Studies 30(1-2): 281-301.

Recibido: 20 de diciembre de 2020

Aceptado: 15 de setiembre de 2021

Maiah Jaskoski es profesora asociada de Northern Arizona University. Autora de Military Politics and Democracy in the Andes (JHUP 2013) y The Politics of Extraction: Territorial Rights, Participatory Institutions, and Conflict in Latin America (OUP, a publicarse), y co-autora y coeditora de American Crossings: Border Politics in the Western Hemisphere (JHUP 2015). Ha publicado artículos en Armed Forces and Society, Comparative Politics, Latin American Politics and Society, Latin American Research Review, Studies in Comparative International Development, Terrorism and Political Violence, World Development. Mail: maiah.jaskoski@nau.edu 
